\title{
Differentiated Thyroid Gland Carcinoma 45 Years and Older AJCC v7 Stage
}

National Cancer Institute

\section{Source}

National Cancer Institute. Differentiated Thyroid Gland Carcinoma 45 Years and Older A/CC v7 Stage. NCI Thesaurus. Code C140963.

A differentiated thyroid gland carcinoma stage for patients 45 years and older defined according to the AJCC 7th edition criteria. 